\title{
Supporting Information for: Liquid Structure and Transport Properties of the Deep Eutectic Solvent Ethaline
}

Yong Zhang, ${ }^{\dagger}$ Derrick Poe, ${ }^{\dagger}$ Luke Heroux ${ }^{\ddagger}$ Henry Squire, ${ }^{\uparrow}$ Brian W. Doherty, ${ }^{\S}$

Zhuoran Long, ${ }^{\S}$ Mark Dadmun, ${ }^{\ddagger} \|$ Burcu Gurkan, $₫$ Mark E. Tuckerman, ${ }^{\S, \perp, \#}$ and Edward J. Maginn*,†

$\dagger$ Department of Chemical and Biomolecular Engineering, University of Notre Dame, Notre Dame, IN 46556 USA

$\ddagger$ Department of Chemistry, University of Tennessee, Knoxville, TN 37996 USA

\Department of Chemical and Biomolecular Engineering, Case Western Reserve University, Cleveland, Ohio 44106

$\S$ Department of Chemistry, New York University, New York, NY 10012 USA

|| Oak Ridge National Laboratory, Chemical Sciences Division, Oak Ridge, TN 37831

$\perp$ Courant Institute of Mathematical Science, New York University, New York, NY 10012 USA

\#NYU-ECNU Center for Computational Chemistry at NYU Shanghai, 3663 Zhongshan Rd. North, Shanghai 200062, China

E-mail: ed@nd.edu 


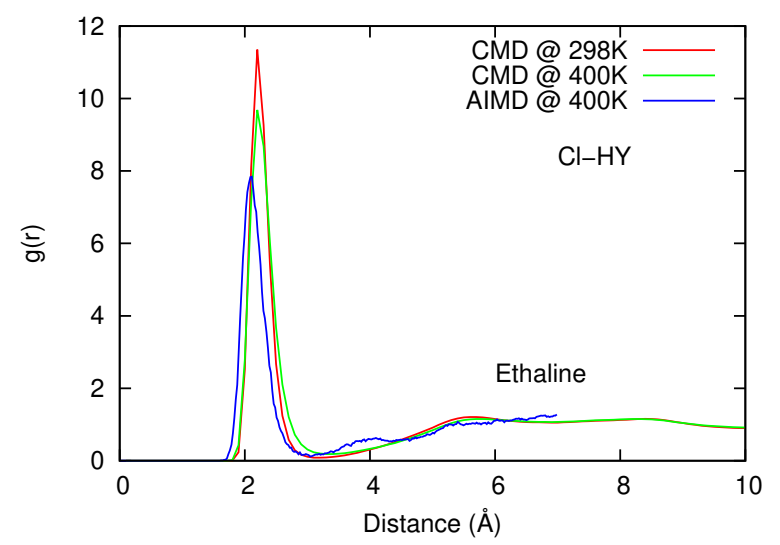

(a)

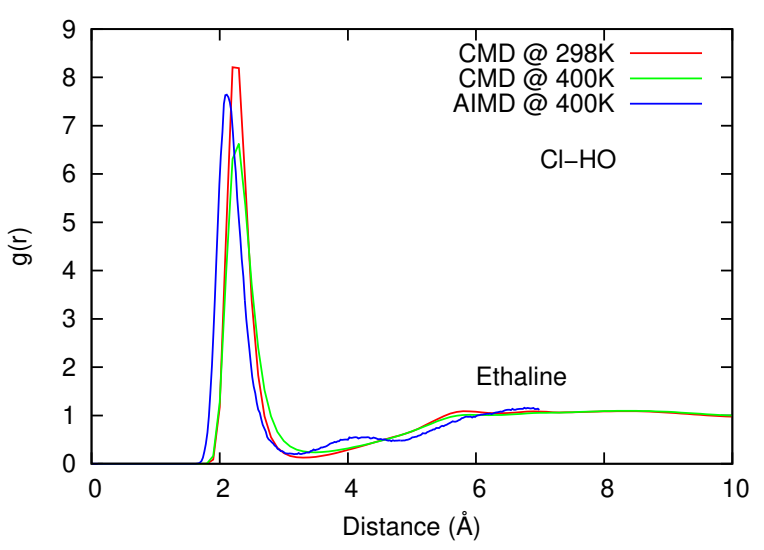

(c)

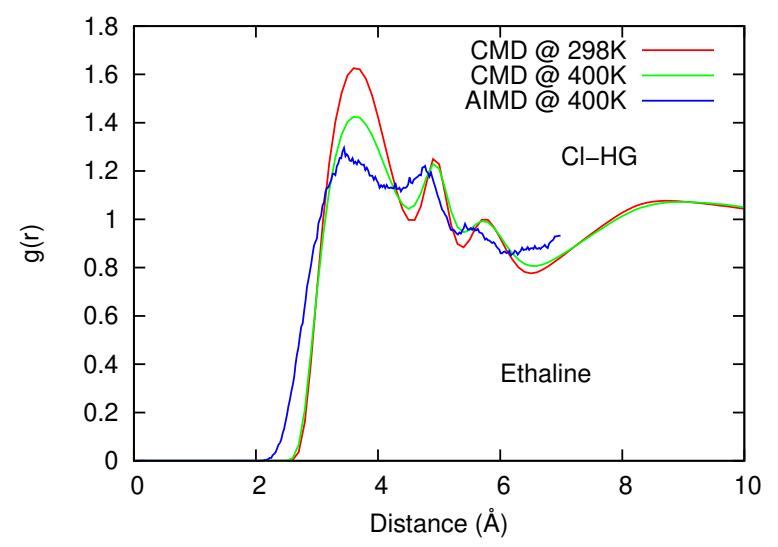

(e)

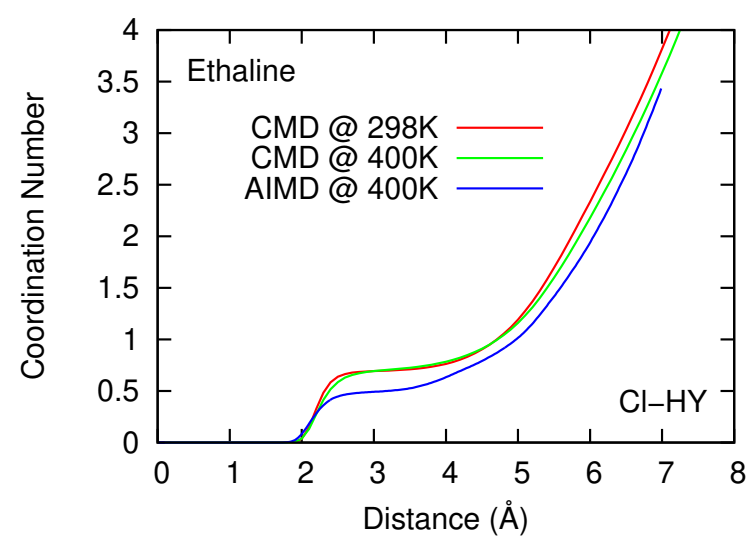

(b)

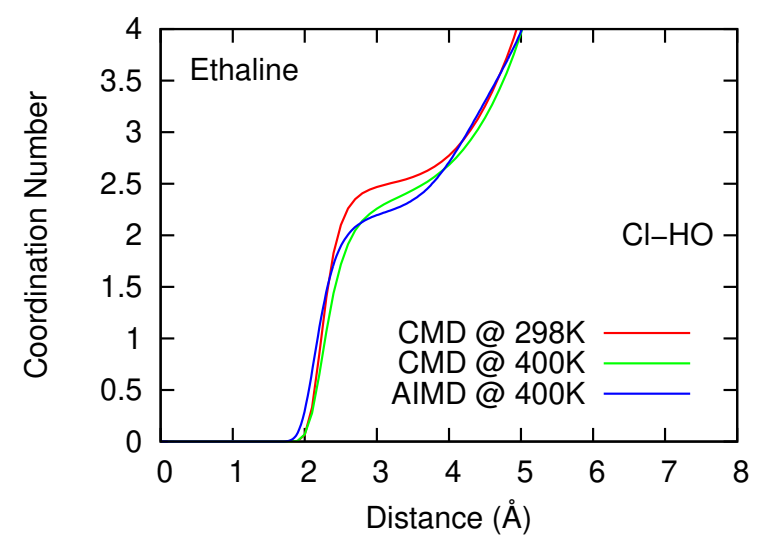

(d)

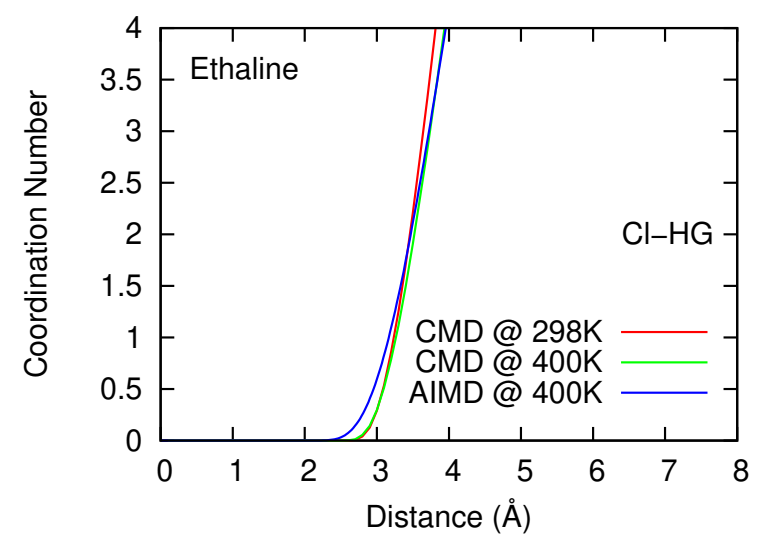

(f)

Figure S1: Comparison of CMD and AIMD partial distribution functions and coordination numbers involving $\mathrm{Cl}$. CMD results at both $298 \mathrm{~K}$ and $400 \mathrm{~K}$ are provided. 


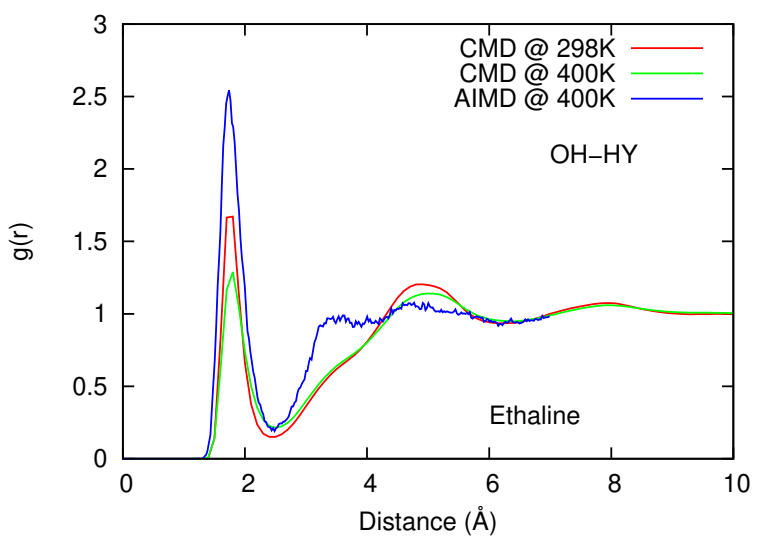

(a)

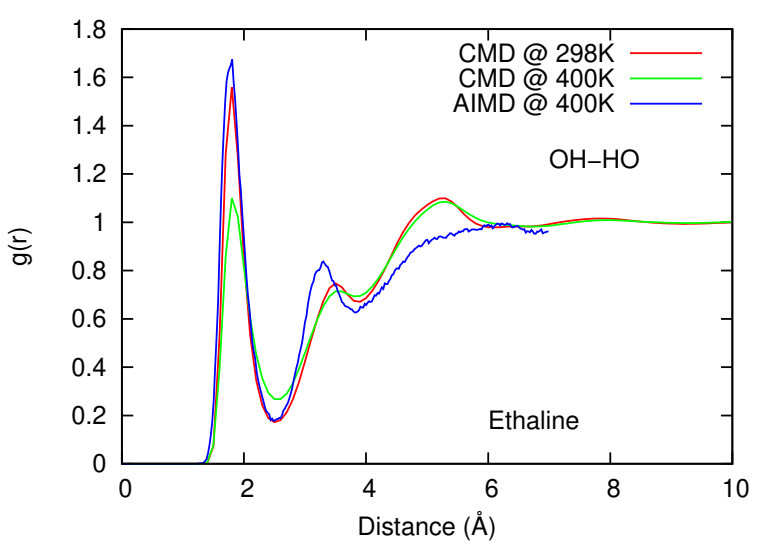

(c)

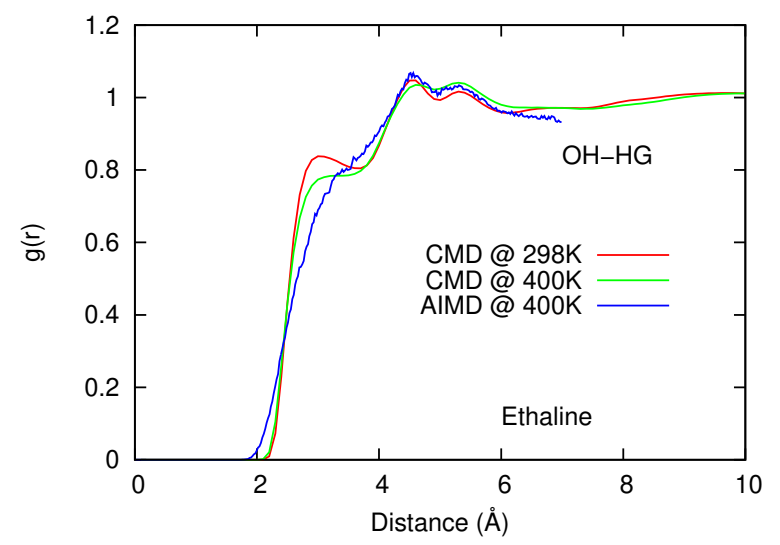

(e)

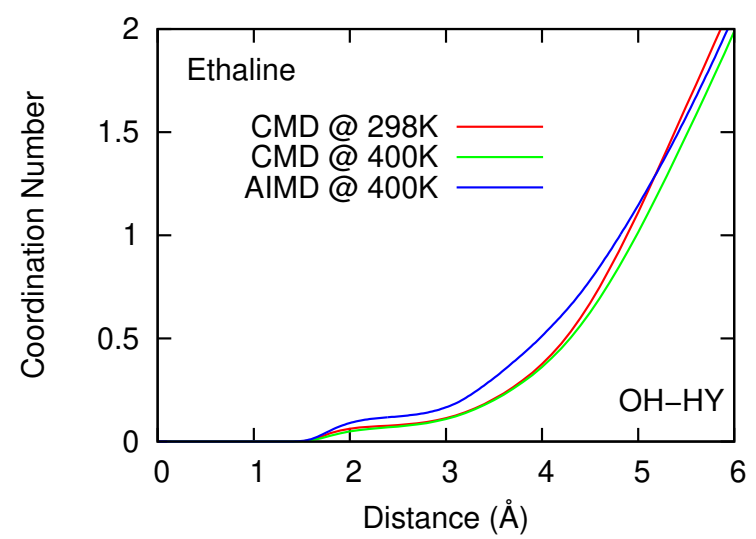

(b)

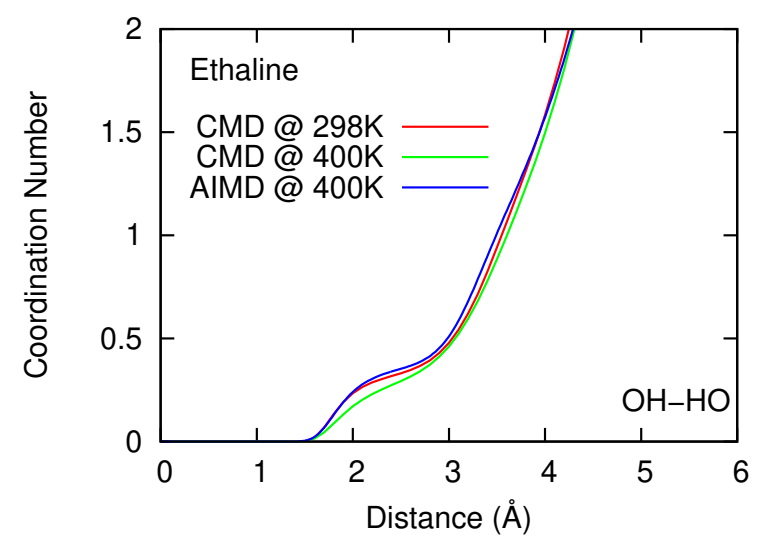

(d)

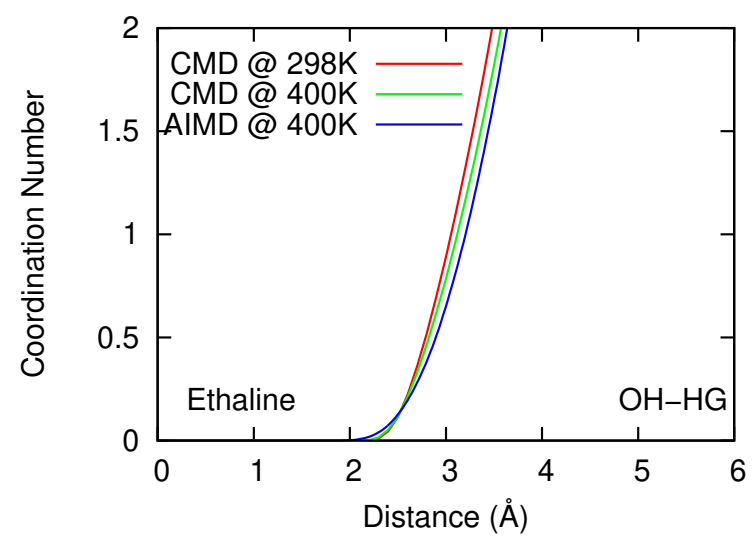

(f)

Figure S2: Comparison of CMD and AIMD partial distribution functions and coordination numbers involving $\mathrm{OH}$ atoms in EG. CMD results at both $298 \mathrm{~K}$ and $400 \mathrm{~K}$ are provided. 


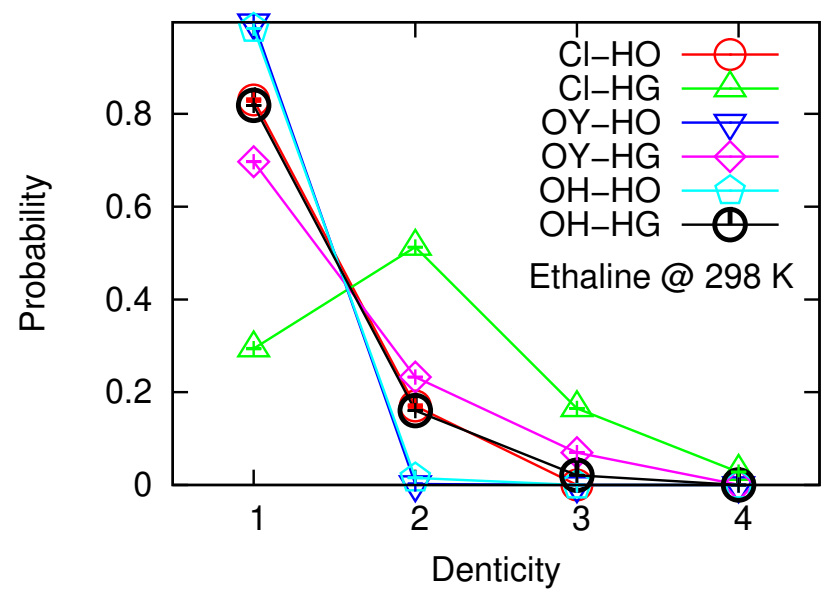

Figure S3: Calculated denticity probability based on CMD simulations at $298 \mathrm{~K}$. 

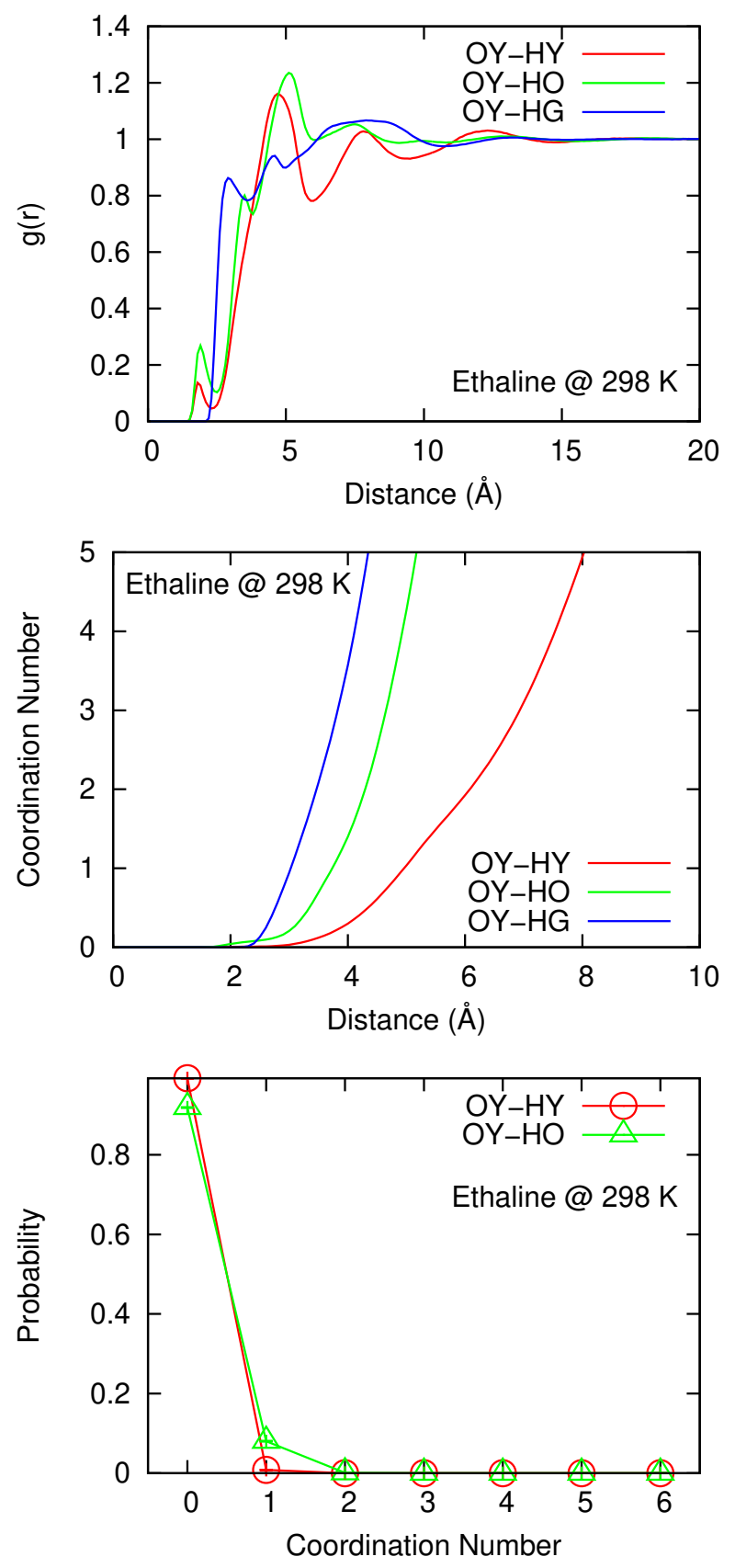

Figure S4: Computed radial distribution functions for a central OY atom of Ch with respect to three different H-groups (a), coordination number as a function of distance about a central OY atom (b), the probability of different numbers of coordinating H-groups about a central OY atom (c) from a CMD simulation at $298 \mathrm{~K}$. 


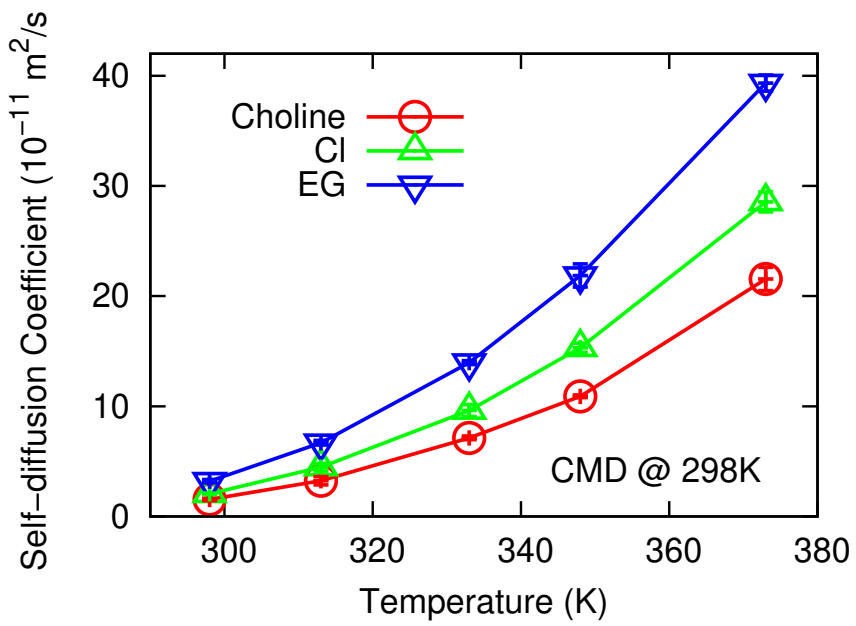

Figure S5: Calculated self-diffusivity as a function of temperature in Ethaline.

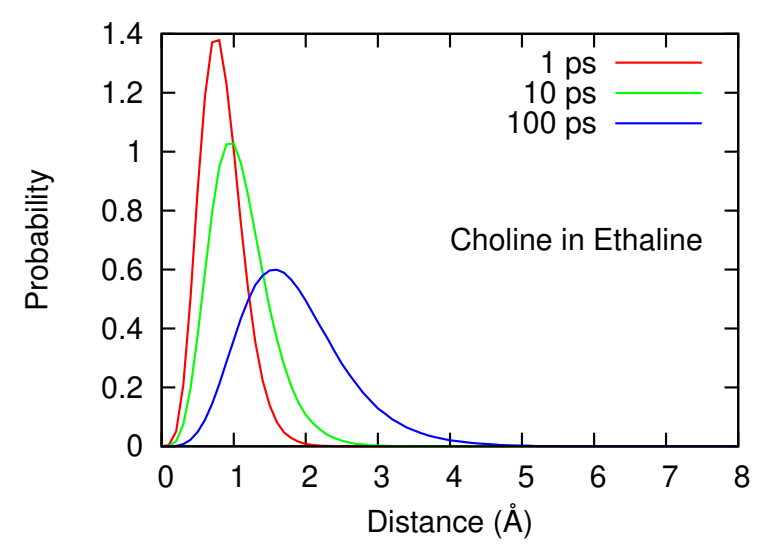

(a)

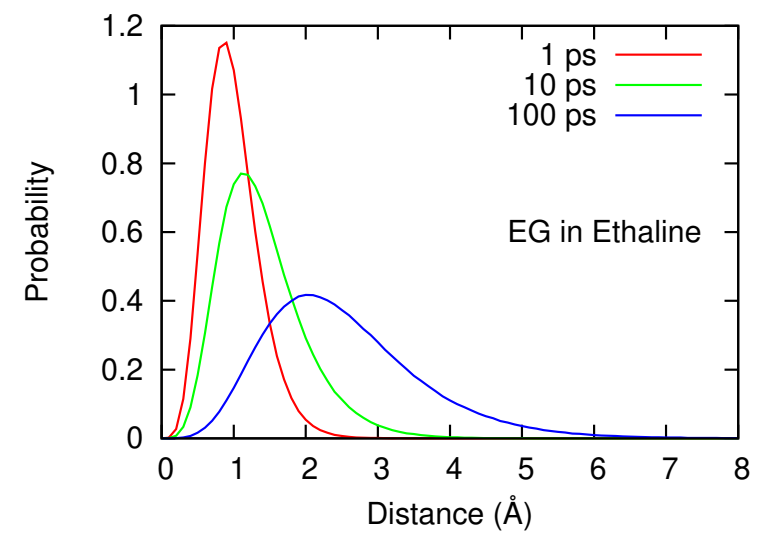

(c)

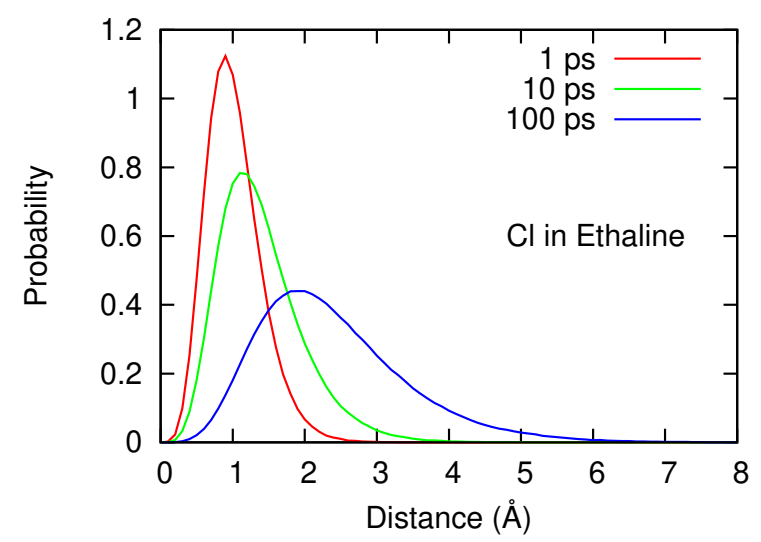

(b)

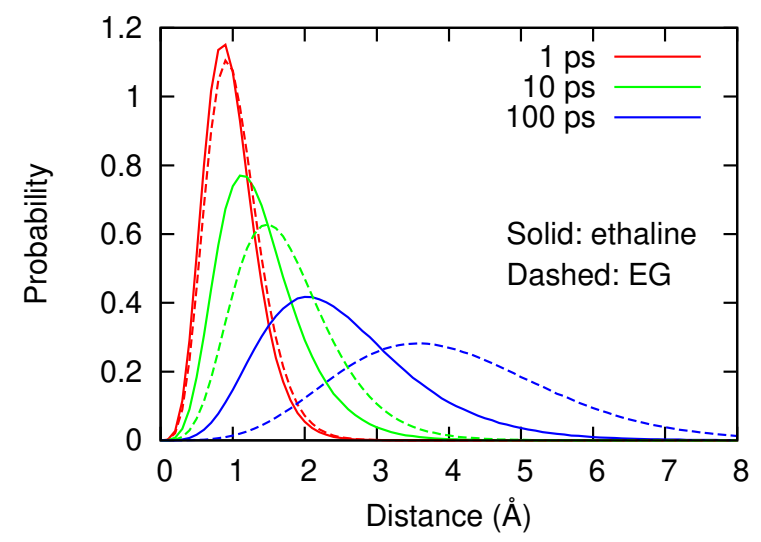

(d)

Figure S6: The self part of the calculated van Hove correlation function based on CMD simulations at $298 \mathrm{~K}$. 

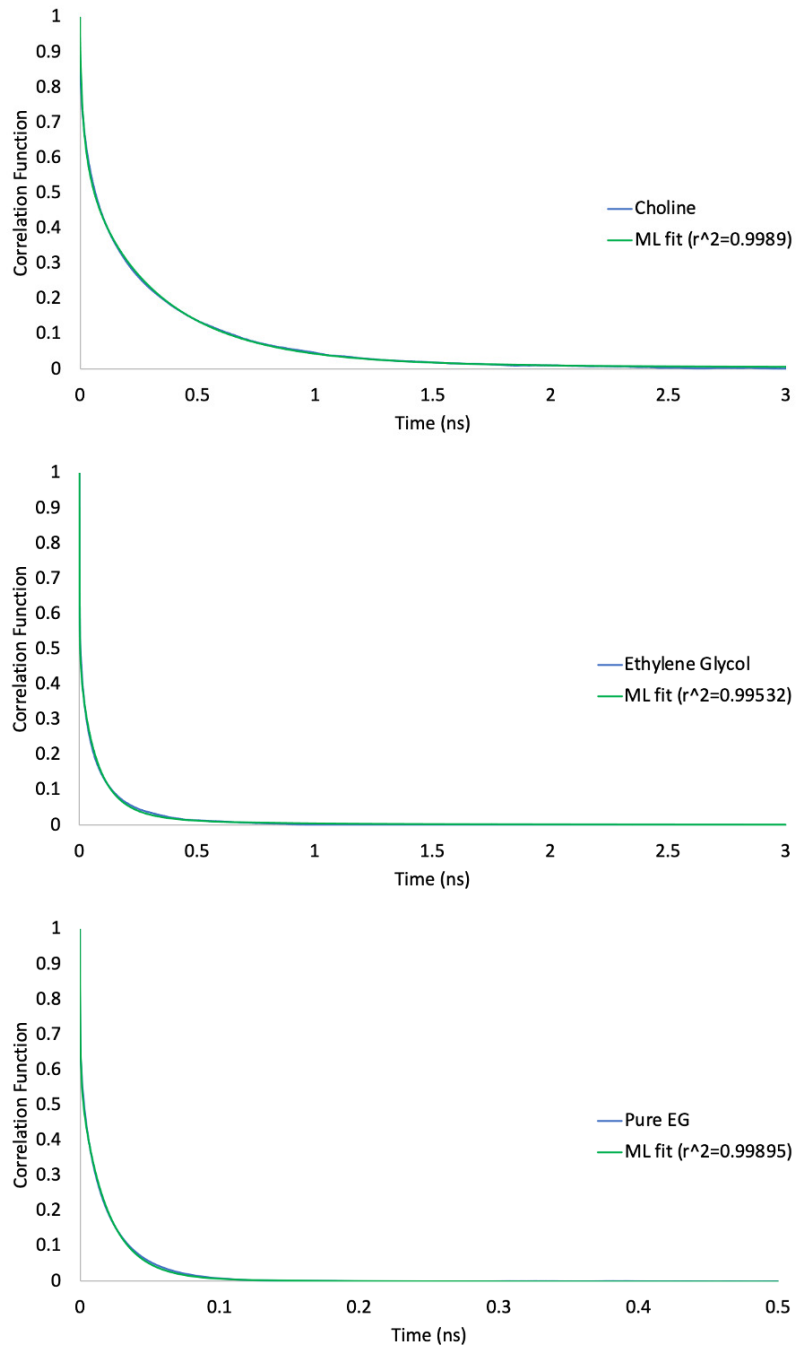

Figure S7: The fitting results of the dipole moment rotational correlation function to the Mittag-Leffler function for choline (top) and EG (middle) in Ethaline and EG in pure EG (bottom). 